\title{
HYDRODYNAMIC AND HYDROMAGNETIC STABILITY OF TWO SUPERPOSED ELASTICO - VISCOUS FLUIDS OF DIFFERENT PERMEABILITY WITH DUST IN POROUS MEDIUM
}

\author{
M. SINGH* \\ Department of Mathematics \\ Govt. Post Graduate College Seema (Rohru), Distt Shimla \\ (H.P) -171207, INDIA \\ E-mail: mahinder_singh91@rediffmail.in \\ C.B. MEHTA \\ Department of Mathematics, Centre for Excellence \\ Govt. Degree College Sanjauli, Distt Shimla \\ (H.P) -171006, INDIA
}

\begin{abstract}
Rayleigh-Taylor instability of two superposed Walters' B has elastico-viscous fluids in a uniform magnetic field through a porous medium with different permeability been studied to include the suspended (dust) particles effect. Using normal mode technique a dispersion relation has been derived. The stability analysis has been carried out. The magnetic field stabilizes the unstable configuration for the wave number band $K>K^{*}$ in which the system is unstable in the absence of the magnetic field. It is also found that for a potential stable arrangement for Walters B' elastico-viscous fluids of different permeabilities in the presence of suspended particles through a porous medium the system is stable, whereas in the potentially unstable case instability of the system occurs.
\end{abstract}

Key word: Rayleigh-Taylor instability, magnetic filed, suspended particle, Walters' B fluid, different permeability and porous medium.

\section{Introduction}

The influence of viscosity on the stability of a plane interface separating two incompressible superposed conducting fluids of uniform densities, when the whole system is acted on by a uniform magnetic field, was studied by Bhatia (1974). Chandra (1938) observed a contradiction between the theory for the onset of convection in fluids heated from below and his experiments. He performed the experiment in an air layer and found that the instability depended on the depth of the layer. A Bénard-type cellular convection with the fluid descending at the cell centre was observed when the predicted gradients were imposed, for layers deeper than $10 \mathrm{~mm}$. A convection which was different in character from that in deeper layers occurred at a much lower temperature gradient than predicted, if the layer depth was less than $7 \mathrm{~mm}$ was called 'columnar instability'. An aerosol was added to mark the flow pattern.

Scanlon and Segel (1973) studied the effect of suspended particles on the onset of Bénard convection and found that the critical Rayleigh number was reduced solely because the heat capacity of the pure gas was supplemented by that of the particles. Sharma et al. (2002) studied the effect of suspended particles on the onset of Bénard convection in hydromagnetics. The effect of suspended particles was found to destabilize the layer whereas the effect of the magnetic field was stabilizing. Walters (1962) reported that the mixture of polymethyl methacrynate and pyridine at $25^{\circ} \mathrm{C}$ containing $30.5 \mathrm{~g}$ of polymer per litre with density $0.98 \mathrm{~g}$ per litre behaves very nearly as Walters (Model B') elastico-viscous fluid. Polymers are used in the manufacture

\footnotetext{
* To whom correspondence should be addressed
} 
of spacecrafts, aeroplanes, tyres belts, conveyer, ropes, cushion seats foam, plastics, engineering equipment, contact lens.

Generally, the magnetic field has a stabilizing effect on the instability but there are a few exceptions. For example, Kent (1966) studied the effect of a horizontal magnetic field, which varies in the vertical direction, on the stability of parallel flows and showed that the system is unstable under certain conditions, while in the absence of the magnetic field the system is known to be stable. Sunil et al. (2006) studied two superposed stratified Walters (Model B') fluids in the presence of a horizontal magnetic field and rotation, and found that the system is unstable for stable stratification for low values of permeability or high values of kinematic viscoelasticity. Kumar et al. (2006) studied MHD instability of rotating superposed Walters' B viscoelastic fluids through a porous medium, and found that the system is stable for low values of permeability or high values of kinematic viscoelasticity. Two superposed Walters B' fluids with suspended particles were studied by Kumar and Singh (2007) They found that the system is stable or unstable depending on the kinematic viscoelasticity, permeability and porosity of the medium. Singh and Kumar (2011) studied hydrodynamic and hydromagnetic stability of two stratified Walters' B elastico-viscous superposed fluids, and found that the system is stable as well as unstable, however for unstable stratifications, the presence of the magnetic field system stabilizes certain wave - number bands, whereas the system is unstable for all wave numbers in the absence of the magnetic field.

Due to a growing importance of the effect of suspended particles on the stability of superposed fluids in the field of industrial and chemical engineering, Sharma (1976) studied the thermal instability of a layer of Oldroydian viscoelastic fluid acted on by a uniform rotation, whereas Sharma and Sharma (1977) studied the instability of the plane interface separating two Oldroydian viscoelastic superposed fluids of uniform densities.

In all the above studies, the fluids have been considered to be Newtonian or viscoelastic (Maxwellian or Oldroydian). There are many elastico-viscous fluids that cannot be characterized by Maxwell's or Oldroyd's constitutive relations. The study of the flow of non-Newtonian fluids having a uniform distribution of dust particles such as latex particles in emulsion paints, reinforcing particles in polymer melts and rock crystals in molten lava is quite useful.

The present study attempts to study the hydrodynamic and hydromagnetic stability of superposed Walters B elastico-viscous fluids permeated with suspended particles through a porous medium.

\section{Formulation of the problem and perturbed equations}

A Walters B' fluid is one of those elastico-viscous fluids which cannot be characterized by Maxwell's constitutive relations or Oldroyd's constitutive relations. As we know, when the fluid permeates a porous material, the gross effect is represented by Darcy's law. As a result of this macroscopic law, the usual viscous and visco-elastic terms in the equation of Walters' B fluid motion are replaced by the resistance term $\left[-\frac{1}{k_{l}}\left(\mu-\mu^{\prime} \frac{\partial}{\partial t}\right) \boldsymbol{q}\right]$. Consider a static state in which an incompressible elastico- viscous fluid-particle layer of variable density is arranged in horizontal strata in a variable porous medium and the pressure $p$, density $\rho$, viscosity $\mu$, suspended particles number density $N$ are functions of the vertical coordinate $z$ only. The character of the equilibrium of this initial static state is determined as usual, by supposing that the system is slightly disturbed and then by following its further evolution.

Let $\rho, p$ and $\boldsymbol{q}(u, v, w)$ denote respectively the density, pressure and filter velocity of the pure fluid; $\boldsymbol{q}_{d}(x, t)$ and $N(x, t)$ denote the velocity and number density of the suspended particles, $\boldsymbol{q}_{d}=(l, r, s), \bar{x}=(x$, $y, z)$ and $\lambda=(0,0,1)$, respectively. $K=6 \pi \rho \vee \eta$ ( $\eta$ the particle radius) is the Stokes' drag coefficient. Let $\in$, $k_{l}, \mu, \mu^{\prime}$ and $\mathrm{g}$ stand for medium porosity, medium permeability, viscosity of the fluid, viscoelasticity of the fluid and acceleration due to gravity respectively. Then the equations of motion and continuity for Walters' B viscoelastic fluid permeated with suspended particles through a porous medium, (Scanlon and Segal, 1973; Sharma and Kumar, 1997) are 


$$
\begin{aligned}
& \frac{\rho}{\epsilon}\left[\frac{\partial \boldsymbol{q}}{\partial t}+\frac{1}{\epsilon}(\boldsymbol{q} \cdot \nabla) \boldsymbol{q}\right]=-\nabla p-\rho g \lambda+\frac{K N}{\epsilon}\left(\boldsymbol{q}_{d}-\boldsymbol{q}\right)-\frac{1}{k_{l}}\left(\mu-\mu^{\prime} \frac{\partial}{\partial t}\right) \boldsymbol{q}, \\
& \nabla \cdot \boldsymbol{q}=0 .
\end{aligned}
$$

Assuming a uniform particle size, spherical shape and small relative velocities between the fluids and particles of two phases, then the net effect of the particles on the fluid through a porous medium is equivalent to an extra body force term per unit volume $\frac{K N}{\epsilon}\left(\boldsymbol{q}_{d}-\boldsymbol{q}\right)$. Since the force exerted by the fluid on the particles is equal and opposite to that exerted by the particles on the fluid, there must be an extra force term, equal in magnitude but opposite in sign, in the equations of motion of the particles. The distances between particles are assumed to be so large compared with their diameter that inter-particle reactions need not be accounted for. The effects of pressure, gravity and Darcian force on the suspended particles, assuming large distances apart, are negligibly small and therefore ignored. If $m N$ is the mass of particles per unit volume, then the equation of motion and continuity for the particles, under the above assumptions, are

$$
\begin{aligned}
& m N\left[\frac{\partial \boldsymbol{q}_{d}}{\partial t}+\frac{1}{\epsilon}\left(\boldsymbol{q}_{d} \cdot \nabla\right) \boldsymbol{q}_{d}\right]=K N\left(\boldsymbol{q}-\boldsymbol{q}_{d}\right), \\
& \in \frac{\partial N}{\partial t}+\nabla \cdot\left(N \boldsymbol{q}_{d}\right)=0 .
\end{aligned}
$$

Since the density of a fluid particle moving with the fluid remains unchanged, we have

$$
\in \frac{\partial \rho}{\partial t}+(\boldsymbol{q} \cdot \nabla) \rho=0
$$

Let $\delta \rho, \delta p, \boldsymbol{q}(u, v, w)$ and $\boldsymbol{q}_{d}(l, r, s)$ denote respectively the perturbations in density $\rho$, pressure $p$, fluid velocity $(0,0,0)$ and particle velocity $(0,0,0)$. Then the linearized perturbation equations of Walters' B' fluid-particle layer are

$$
\begin{aligned}
& \frac{\rho}{\in} \frac{\partial \boldsymbol{q}}{\partial t}=-\nabla \delta p-g \delta \rho \lambda+\frac{K N}{\epsilon}\left(\boldsymbol{q}_{d}-\boldsymbol{q}\right)-\frac{1}{k_{l}}\left(\mu-\mu^{\prime} \frac{\partial}{\partial t}\right) \boldsymbol{q} \\
& \nabla \cdot \boldsymbol{q}=0 \\
& \left(\frac{m}{K} \frac{\partial}{\partial t}+1\right) \boldsymbol{q}_{d}=\boldsymbol{q} \\
& \in \frac{\partial}{\partial t} \delta \rho=-w(D \rho), \\
& \frac{\partial M}{\partial t}+\nabla \cdot \boldsymbol{q}_{d}=0
\end{aligned}
$$


where $M=\frac{\in N}{N_{0}}$ and $N_{0}, N$ stand for the initial uniform number density and perturbation in number density, respectively and $D=\frac{d}{d z}$.

Analyzing the disturbances into normal modes, we seek solutions whose dependence on $x, y$ and $t$ is given by

$$
e^{\left(i k_{x} x+i k_{y} y+n t\right)}
$$

where $n$ is, in general, a complex constant. $k_{x}, k_{y}$ are wave numbers along $x$ - and $y$-directions and $k^{2}\left(=k_{x}^{2}+\right.$ $\left.k_{y}^{2}\right)$.

For perturbation solutions in the form Eq.(2.11); after elimination of Eqs (2.6)-(2.9), $\boldsymbol{q}_{d}$, yield

$$
\begin{aligned}
& \frac{\rho}{\epsilon} n u=-i k_{x} \delta p-\frac{1}{k_{l}}\left(\mu-\mu^{\prime} n\right) u-\frac{m N}{\in(\tau n+1)} n u, \\
& \frac{\rho}{\epsilon} n v=-i k_{y} \delta p-\frac{1}{k_{l}}\left(\mu-\mu^{\prime} n\right) v-\frac{m N}{\epsilon(\tau n+l)} n v, \\
& \frac{\rho}{\epsilon} n w=-D \delta p-\frac{1}{k_{l}}\left(\mu-\mu^{\prime} n\right) w-\frac{m N}{\in(\tau n+l)} n w-g \delta \rho, \\
& i k_{x} u+i k_{y} v+D w=0, \\
& \in n \delta \rho=-(D \rho) w
\end{aligned}
$$

where $\tau=\frac{m}{K}$. Eliminating $\delta p$ between Eqs (2.12)-(2.14) and using Eqs (2.15) and (2.16), we obtain

$$
\begin{aligned}
& n\left[D\left(\frac{\rho}{\epsilon} D w\right)-\frac{k^{2}}{\epsilon} \rho w\right]+\left[D\left\{\left(\frac{\mu}{k_{l}}-\frac{\mu^{\prime}}{k_{l}} n\right) D w\right\}-k^{2}\left(\frac{\mu}{k_{l}}-\frac{\mu^{\prime}}{k_{l}} n\right) w\right]+ \\
& +\frac{n}{\in(\tau n+1)}\left\{D[m N D w]-k^{2} m N w\right\}=-\frac{g k^{2}}{\in n}(D \rho) w .
\end{aligned}
$$

\section{Two uniform fluids separated by a horizontal boundary}

Consider the case of two uniform Walters B' viscoelastic fluids of densities, viscosities, viscoelasticities, suspended particles number densities as $\rho_{2}, \mu_{2}, \mu_{2}^{\prime}, N_{2}$ and $\rho_{1}, \mu_{1}, \mu_{1}^{\prime}, N_{1}$ separated by a horizontal boundary at $z=0$. The subscripts 1 and 2 distinguish the lower and the upper fluids, respectively. The medium porosity $\in$ is assumed to be the same in both the regions. Let the medium permeabilities of upper $(z>0)$ and lower $(z<0)$ media be $k_{12}$ and $k_{11}$, respectively. reduces to

Then in each region of constant $\rho$, constant $\mu$, constant $\mu^{\prime}$, constant $N$ and constant $K_{l}$ Eq.(2.17)

$$
\left(D^{2}-k^{2}\right) w=0 .
$$




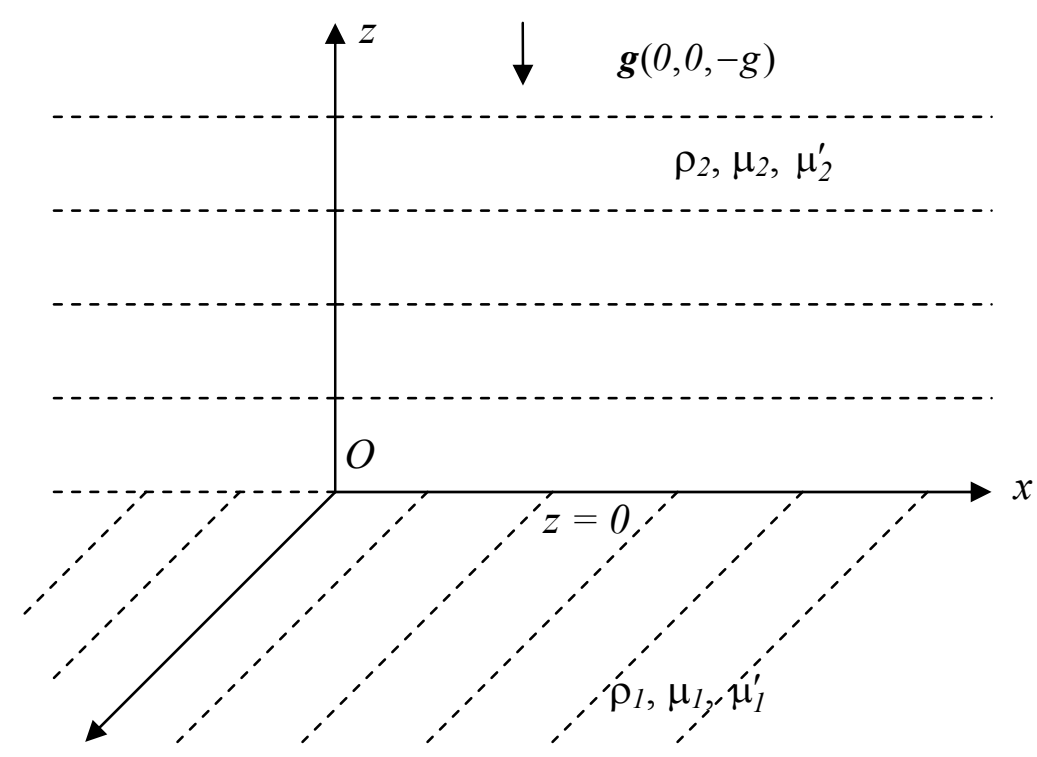

Fig.1. Two uniform Walters B' elastico-viscous fluids separated by a horizontal boundary.

The general solution of Eq.(2.18) is

$$
w=A e^{+k z}+B e^{-k z}
$$

where $A$ and $B$ are arbitrary constants. Then the boundary conditions to be satisfied in the present problem are

(i) The velocity $w \rightarrow 0$ when $z \rightarrow \infty$ (for the upper fluid) and $z \rightarrow-\infty$ (for the lower fluid).

(ii) $\quad w(z)$ is continuous at $z=0$.

(iii) The jump condition at the interface $z=0$ between the fluids. is obtained by integrating Eq.(2.17) across the interface $z=0$ and is

$$
\begin{aligned}
& \frac{n}{\epsilon}\left(\rho_{2} D w_{2}-\rho_{1} D w_{1}\right)_{z=0}+\left[\left(\frac{\mu_{2}}{k_{12}}-\frac{\mu_{2}^{\prime}}{k_{12}} n\right) D w_{2}-\left(\frac{\mu_{1}}{k_{11}}-\frac{\mu_{1}^{\prime}}{k_{11}} n\right) D w_{1}\right]_{z=0}+ \\
& +\frac{m n}{\in(\tau n+1)}\left[N_{2} D w_{2}-N_{1} D w_{1}\right]_{z=0}=-g \frac{k^{2}}{\in n}\left(\rho_{2}-\rho_{1}\right) w_{0}
\end{aligned}
$$

where $w_{0}$ is the common value of $w$ at $z=0$.

Applying boundary conditions (i) and (ii), we can write

$$
\begin{aligned}
& w_{1}=A e^{k z}, \quad(z<0), \\
& w_{2}=A e^{-k z}, \quad(z>0)
\end{aligned}
$$

where the same constant $A$ has been considered to ensure the continuity of $w$ at $z=0$. Applying condition (3.3) to solutions (3.4) and (3.5), we obtain 


$$
\begin{aligned}
& \tau\left[\frac{\alpha_{2}+\alpha_{1}}{\epsilon}-\left(\frac{\alpha_{2} v_{2}^{\prime}+\alpha_{1} v^{\prime}}{K_{12}-K_{11}}\right)\right] n^{3}+\left[\frac{1}{\epsilon}+\frac{m}{\epsilon}\left(\frac{N_{2}+N_{1}}{e_{2}+e_{1}}\right)+\tau\left(\frac{\alpha_{2} u_{2}}{k_{12}}+\frac{\alpha_{1} v_{1}}{k_{11}}\right)-\left(\frac{\alpha_{2} v^{\prime}}{k_{12}}+\frac{\alpha_{1} v_{1}^{\prime}}{k_{11}}\right)\right] n^{2}+ \\
& +\left[\frac{\alpha_{2} v_{2}}{k_{12}}+\frac{\alpha_{1} v_{1}}{k_{11}}-g \frac{k \tau}{\epsilon}\left(\alpha_{2}-\alpha_{1}\right)\right] n-g \frac{k}{\epsilon}\left(\alpha_{2}-\alpha_{1}\right)=0
\end{aligned}
$$

where $v_{1,2}=\frac{\mu_{1,2}}{\rho_{1,2}}, v_{1,2}^{\prime}=\frac{\mu_{1,2}^{\prime}}{\rho_{1,2}^{\prime}}$, and $\alpha_{1,2}=\frac{\rho_{1,2}}{\rho_{1}+\rho_{2}}$. So $\alpha_{1}+\alpha_{2}=1$.

\section{Discussion}

(a) Stable case $\left(\alpha_{2}<\alpha_{1}\right)$

For the potentially stable arrangement $\left(\right.$ i.e., $\left.v_{1}^{\prime}<\frac{k_{11}}{\epsilon}, v_{2}^{\prime}<\frac{k_{12}}{\epsilon}\right)$, there is no change in the coefficients of Eq.(3.6), therefore, all the three roots of Eq.(3.6) are either real, negative or there is one real, negative root and the other two are complex conjugates with negative real parts. The system is, therefore, stable in each case. This is in agreement with the stability of Newtonian superposed fluids permeated with suspended particles in a porous medium, where also the system is always stable for the stable arrangement.

However, this is in contrast to the stability of Walters B' elastico -viscous fluids permeated with suspended particles in a porous medium with different permeability and for the potentially stable arrangement where the system is found to be unstable or stable depending on whether the kinematic viscoelasticity is greater or less than the medium permeability divided by medium porosity, in each region.

(b) Unstable case $\left(\alpha_{2}>\alpha_{1}\right)$

For the potentially unstable arrangement, i.e., $\frac{g k \tau}{\epsilon}\left(\alpha_{2}-\alpha_{1}\right)>\frac{\alpha_{2} v_{2}}{k_{12}}+\frac{\alpha_{1} v_{1}}{k_{11}}$ and $\frac{\alpha_{2} v_{2}^{\prime}}{k_{12}}+\frac{\alpha_{1} v_{2}^{\prime}}{k_{11}}>\frac{1}{\epsilon}+$ $+\frac{m}{\epsilon}\left(\frac{N_{2}+N_{1}}{e_{2}+e_{1}}\right)+\tau\left(\frac{\alpha_{2} v_{2}}{k_{12}}+\frac{\alpha_{1} v_{1}}{k_{11}}\right)$, the constant term in Eq.(3.6) is negative therefore equation (3.6) has a change of sign and hence allows one positive root. The occurrence of positive root implies instability of the system.

We, therefore, conclude that for two superposed Walters B' elastico-viscous fluids permeated with suspended particles in a porous medium with different permeability and for the potentially unstable arrangement, the system is unstable. This is in agreement with the stability of Newtonian superposed fluids permeated with suspended particles in a porous medium with variable permeability, where the system is also always unstable for the unstable arrangement.

\section{Effect of a variable horizontal magnetic field}

Here we consider a static state in which an incompressible, infinitely electrically conducting Walters' B' elastico-viscous fluid is arranged in horizontal strata in a porous medium with variable permeability in the presence of suspended particles and a variable horizontal magnetic field $\boldsymbol{H}(H(z), 0,0)$. Let $\boldsymbol{h}\left(h_{x}, h_{y}, h_{z}\right)$ denote the perturbation in the magnetic field, $\mu_{e}$ stands for magnetic permeability. Then the linearized hydromagnetic perturbation equations, relevant to the problem, are 


$$
\begin{aligned}
& \frac{\rho}{\in} \frac{\partial \boldsymbol{q}}{\partial t}=-\nabla \delta p-g \delta \rho \lambda+\frac{k N}{\epsilon}\left(\boldsymbol{q}_{d}-\boldsymbol{q}\right)-\frac{1}{k_{l}}\left(\mu-\mu^{\prime} \frac{\partial}{\partial t}\right) \boldsymbol{q}+ \\
& +\frac{\mu_{e}}{4 \pi}[(\nabla \times \boldsymbol{h}) \times \boldsymbol{H}+(\nabla \times \boldsymbol{H}) \times \boldsymbol{h}], \\
& \nabla \cdot \boldsymbol{h}=0, \\
& \in \frac{\partial \boldsymbol{h}}{\partial t}=\nabla \times(\boldsymbol{q} \times \boldsymbol{H}),
\end{aligned}
$$

together with Eqs (2.7)-(2.9), using Eq.(2.11), eliminating $u, v, h_{x}, h_{y}, h_{z}, \delta \rho$ and $\delta p$ in Eqs (4.1)-(4.3) and Eqs (2.7)-(2.9), we obtain

$$
\begin{aligned}
& n\left[D\left(\frac{\rho}{\epsilon} D w\right)-\frac{k^{2}}{\epsilon} \rho w\right]+\left\{\left[D\left(\frac{\mu}{k_{l}}-\frac{\mu^{\prime}}{k_{l}} n\right) D w\right]-k^{2}\left(\frac{\mu}{k_{l}}-\frac{\mu^{\prime}}{k_{l}} n\right)\right\}+ \\
& +\frac{n}{\in(\tau n+1)}\left[D(m N D w)-k^{2} m N w\right]+\frac{\mu_{e} k_{x}^{2}}{4 \pi n \in}\left[D\left(H^{2} D w\right)-H^{2} k^{2} w\right]=-\frac{g k^{2}}{\in n}(D \rho) w .
\end{aligned}
$$

\section{Two uniform Walters' B visco-elastic fluids separated by a horizontal boundary}

Here we consider the case of two uniform, Walters' B elastico-viscous densities, viscosities, viscoelasticities, suspended particles number densities, magnetic fields as $\rho_{2}, \mu_{2}, \mu_{2}^{\prime}, N_{2}, H_{2}$ and $\rho_{1}, \mu_{1}, \mu_{1}^{\prime}$, $N_{l}, H_{l}$ separated by a horizontal boundary $z=0$ in a porous medium. Let the medium permeabilities of upper $(z>0)$ and lower $(z<0)$ media be $k_{12}$ and $k_{11}$, respectively.

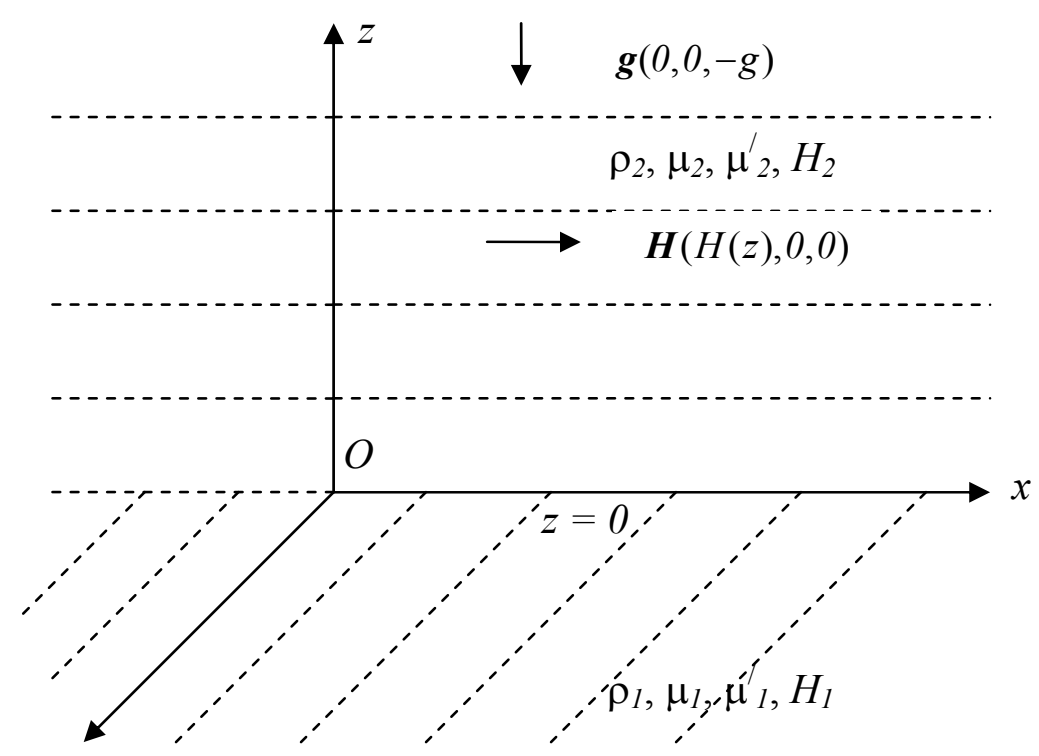

Fig.2. Two uniform Walters' B elastico-viscous fluids separated by a horizontal boundary. 

reduces to

Then in each region of constant $\rho$, constant $\mu$, constant $\mu^{\prime}$, constant $m N$, constant $k_{1}$, Eq.(4.4)

$$
\left(D^{2}-k^{2}\right) w=0 .
$$

The boundary conditions to be satisfied in the present problem are (i) the vanishing of $w$ at $z \rightarrow+\infty$ and $z \rightarrow-\infty$, (ii) continuity of $w$ at $z=0$ and (iii) the jump condition. The jump condition is obtained by integrating (4.4) across the interface at $z=0$ and is

$$
\begin{aligned}
& \frac{n}{\epsilon}\left(\rho_{2} D w_{2}-\rho_{1} D w_{1}\right)_{z=0}+\left[\left(\frac{\mu_{2}}{k_{12}}-\frac{\mu_{2}^{\prime}}{k_{12}} n\right) D w_{2}-\left(\frac{\mu_{2}}{k_{11}}-\frac{\mu_{2}^{\prime}}{k_{11}} n\right) D w_{1}\right]_{z=0}+\frac{m n}{\in(\tau n+l)}+ \\
& {\left[N_{2} D w_{2}-N_{1} D w_{1}\right]_{z=0}+\frac{\mu_{e} k_{x}^{2}}{4 \pi n \epsilon}\left(H_{2}^{2} D w_{2}-H_{1}^{2} D w_{1}\right)_{z=0}=-g \frac{k^{2}}{\in n}\left(\rho_{2}-\rho_{1}\right) w_{0} .}
\end{aligned}
$$

Applying boundary conditions (i) and (ii), the solution of Eq.(5.1) is given by Eqs (3.4) and (3.5), in Eq.(5.2), we obtain

$$
\begin{aligned}
& \tau\left[\left(\frac{\alpha_{2}+\alpha_{1}}{\epsilon}\right)-\left(\frac{\alpha_{1} v_{2}^{\prime}}{k_{12}}+\frac{\alpha_{1} v_{1}^{\prime}}{k_{11}}\right)\right] n^{3}+\left[\frac{\alpha_{1}+\alpha_{2}}{\epsilon}+\frac{m}{\epsilon}\left(\frac{N_{2}+N_{1}}{\rho_{2}+\rho_{1}}\right)+\tau\left(\frac{\alpha_{2} v_{2}}{k_{12}}+\frac{\alpha_{1} v_{1}}{k_{11}}\right)+\right. \\
& \left.-\left(\frac{\alpha_{2} v_{2}^{\prime}}{k_{12}}+\frac{\alpha_{1} v_{1}}{k_{11}}\right)\right] n^{2}+\left[\left(\frac{\alpha_{2} v_{2}}{k_{12}}+\frac{\alpha_{1} v_{1}}{k_{11}}\right)+\frac{\tau k_{x}^{2}}{\epsilon}\left(V_{A_{2}}^{2}-V_{A_{1}}^{2}\right)+\right. \\
& \left.-g \frac{k \tau}{\epsilon}\left(\alpha_{2}-\alpha_{1}\right) n\right]+\left[\frac{k_{x}^{2}}{\epsilon}\left(V_{A_{2}}^{2}+V_{A_{1}}^{2}\right)-\frac{g k}{\epsilon}\left(\alpha_{2}-\alpha_{1}\right)\right]=0
\end{aligned}
$$

where

$$
v_{1,2}=\frac{\mu_{1,2}}{\rho_{1,2}}, v_{1,2}^{\prime}=\frac{\mu_{1,2}^{\prime}}{\rho_{1,2}^{\prime}} \text {, and } \alpha_{1,2}=\frac{\rho_{1,2}}{\rho_{1}+\rho_{2}}, V_{A 2}^{2}=\frac{\mu_{e} H_{2}^{2}}{4 \pi\left(\rho_{1}+\rho_{2}\right)} \text { and } V_{A 1}^{2}=\frac{\mu_{e} H_{1}^{2}}{4 \pi\left(\rho_{2}+\rho_{1}\right)}
$$

\section{Discussions:}

(a) Stable case $\left(\alpha_{2}<\alpha_{1}\right)$

For the potentially stable arrangement, i.e., $\left(v_{2}^{\prime}>\frac{k_{12}}{\epsilon}, v_{1}^{\prime}>\frac{k_{11}}{\epsilon}\right)$, there is no change of sign in the coefficients of Eq.(5.3). The system is, therefore, stable. Therefore, we conclude that for two superposed Walters' B elastico-viscous fluids permeated with suspended particles in a porous medium with variable permeability in hydromagnetics field is instable. The result is the same in the hydrodynamic case, however, this is in contrast to the stability of Walters (model B') superposed fluids permeated with suspended particles in a porous medium, with variable permeability, and the system can be stable or unstable depending on whether the kinematic viscoelasticity is less than or greater than the medium permeability divided by medium porosity, in each region. 
(b) Unstable case $\left(\alpha_{2}>\alpha_{1}\right)$

For the potentially unstable arrangement, i.e., $v_{2}^{\prime}<\frac{k_{12}}{\epsilon}, v_{1}^{\prime}<\frac{K_{11}}{\epsilon}$ the system in stable or unstable according to

$$
k_{x}^{2}\left(V_{A_{1}}^{2}+V_{A_{2}}^{2}\right)>\text { or }<g k\left(\alpha_{2}-\alpha_{1}\right)
$$

i.e., $\quad \rho_{2}-\rho_{1}<$ or $>\frac{\mu_{e} k_{x}^{2}}{4 \pi g k}\left(H_{1}^{2}+H_{2}^{2}\right)$.

The system is clearly unstable in the absence of the magnetic field. However, the system is completely stabilized by a large magnetic field.

$$
\rho_{2}-\rho_{1}<\frac{\mu e k_{x}^{2}}{4 \pi g k}\left(H_{1}^{2}+H_{2}^{2}\right)
$$

The presence of the field thus completely stabilizes the wave number band $k>k^{*}$, where $k^{*}=\frac{4 \pi g\left(\rho_{2}-\rho_{1}\right)}{\mu_{e}\left(H_{1}^{2}+H_{2}^{2}\right)} \sec ^{2} \theta$, Here $\theta$ is the angle between $k_{x}$ and $k$ such that $k_{x}=k \cos \theta$.

\section{Concluding remarks}

The hydrodynamic and hydromagnetic stability of two superposed Walters' (Model B) elastico viscous fluids of different permeability in the presence of suspended particles in a porous medium has been investigated. The main results from the analysis are:

(1): When kinematic viscoelasticity is less than the medium permeability divided by medium porosity, than the system is stable for the potential stable arrangement in the case of suspended particles in a porous medium.

(2): The case of the potential unstable arrangement, for two superposed Walters' (Model B) elastico viscous fluids permeated with suspended particles in a porous medium with different permeability is always unstable.

(3): Using normal mode technique a dispersion relation has been derived.

(4): The magnetic field stabilizes the unstable configuration for wave number band $\mathrm{K}>\mathrm{K}^{*}$, in which the system is unstable in the absence of the magnetic field.

\section{Acknowledgement}

The Authors are thankful to the chief editor and learned referee for his useful technical comments and valuable suggestion, which led to a significant improvement of this paper.

\section{Nomenclature}

$$
\begin{aligned}
g & - \text { acceleration due to gravity } \\
\boldsymbol{H}(H(z), 0,0) & - \text { variable horizontal magnetic field }
\end{aligned}
$$




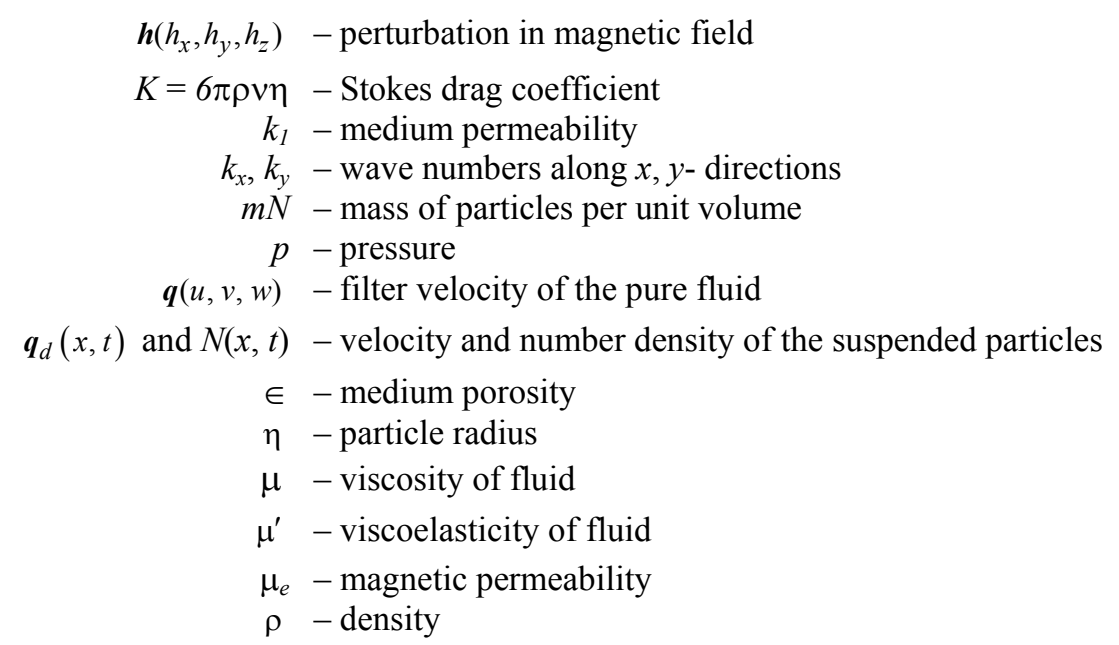

\section{References}

Bhatia P.K. (1974): Rayleigh-Taylor instability of two viscous superposed conducting fluids. - Nuovo Cimento, 19 B pp.161-168.

Chandra K. (1938): Instability of fluid heated from below. - Proc. Roy. Soc. (Lon.), 164A, pp.231-242.

Kent A. (1966): Instability of laminar flow of a magneto-fluid. - Phys. Fluids., vol.9, pp.1286-1289.

Kumar P., Lal R. and Singh G.J. (2006): MHD instability of rotating superposed Walter's B' viscoelastic fluids through a porous medium. - Journal of Porous Media, vol.9, No.5, pp.463-468.

Kumar P. and Singh M. (2007): instability of two rotating viscoelastic (Walter's B') superposed fluids with suspended particles in porous medium. - Thermal Science, vol.11, No.1, pp.93-102.

Scanlon J.W. and Segel L.A. (1973): Some effects of suspended particles on the onset of Bénard convection. - Phys. Fluids., vol.16, pp.1573-1578.

Sharma R.C. (1976): Effect of rotation on thermal instability of a viscoelastic fluid. - Acta Physica Hungarica, vol.40, pp.11-17.

Sharma R.C. and Kumar P. (1997): Hydromagnetic stability of two Rivlin-Ericksen elastico-viscous superposed conducting fluids. - Z. Naturforsch., vol.52a, pp.528-532.

Sharma R.C., Kumar P. and Sharma S. (2002): Rayleigh-Taylor instability of Walter's B' elastico - viscous fluid through porous medium. - Int. J. of Applied Mechanics Engineering, vol.7, No.2, pp.433-444.

Sharma R.C. and Sharma K.C. (1977): Rayleigh-Taylor instability of two superposed conducting fluids in the presence of suspended particles. - Acta Physica Hungarica, vol.43, pp.251-258.

Singh M. and Kumar P. (2011): Hydrodynamic and hydromagnetic stability of two stratified Walter's B' elastico viscous superposed fluids. - Int. J. of Applied Mechanics and Engineering, vol.16, No.1, pp.233-241.

Sunil, Gupta U. and Sharma V. (2006): Stability of stratified elastico - viscous Walter's (model B') fluid in the presence of horizontal magnetic field and rotation in porous medium. - Arch. Mech., vol.58, No.1, pp.187-197.

Walters K. (1962): Non-Newtonian effects in some elastico-viscous liquids whose behaviour at small rates of shear is characterized by a general linear equation of state. - Quart. J. Mech. Applied Math., vol.15, No.1, pp.63-76. 